\title{
Application of Multiple Views and Stereo-3D Imaging Technology to Burn Care
}

\author{
Tung Jing FANG ${ }^{* 1,2,3}$, Chen HAN ${ }^{4}$, Lai Chung LEE ${ }^{4}$, Chuan Chia WANG ${ }^{5}$, Chia Chung FANG ${ }^{6}$ \\ ${ }^{1}$ Department of Electronic Engineering, National Taipei University of Technology, Taipei, Taiwan; \\ 2 Department of Internal Medicine, Taipei Veterans-General Hospital and \\ Tri-Service General Hospital, Taipei, Taiwan; \\ ${ }^{3}$ Department of Physiology and Biophysics, Institute of Physiology, \\ National Defense Medical Center, Taipei, Taiwan; \\ ${ }^{4}$ Institute of Interaction Design, National Taipei University of Technology, Taipei, Taiwan; \\ ${ }^{5}$ GIGA-BYTE Technology Co., Ltd., Taipei, Taiwan; \\ ${ }^{6}$ Lab of Stem Cell and Tissue Regeneration, National Defense Medical Center, Taipei, Taiwan
}

https://doi.org/10.15221/20.16

\begin{abstract}
Objectives: This study is concerned with the geometrical part of the vision, or more specifically with the $3 \mathrm{D}$ aspects. The ability of humans to perceive the $3 \mathrm{D}$ world from the two-dimensional (2D) projections on the retinae is fascinating. How some of the basic sub-problems of geometric vision can be solved, but the overall aim was to reconstruct a scene from several images. So how do we combine the basic solvers to handle multiple images?

Methods: We studied the multiple views and stereo-3D imaging technology to burn injury of stereo 3D vision and $3 \mathrm{D}$ studied visual issues related to it, including visual discomfort, binocular disparities, vengeance-accommodation mismatch, and visual fatigue. We also studied the burn care of medical applications of stereo-3D imaging techniques, and application trends as pertain to imaging medical devices. A new camera having many correspondences among the estimated 3D points is added to the model by computing its camera pose. To stabilize the estimation bundle adjustment is normally performed several times, sometimes for every new camera that is added. T The Face \& Future SID® device is a mobile 3D wound measurement device attached to an App It has a friendly user interface and a LED assisted multiple views 3D camera that can assess both wound area and volume. In this study, we compare three modalities in wound area measurements: The Face \& Future SID $B$ device, the device, and standard hand measurements.

Results: The multiple views 3D face imaging technology, we are providing the convenience and widespread use for 3 main groups in the catastrophe inclusive of patients, emergency medical technicians (EMTs), and ambulance technicians and doctors. For patients who could be civilians or our soldiers who participate in the rescue mission, the 3D face imaging device could lead to developing new dressing for the wound on the face. The new dressing could fully cover the wound and prevent the production of dead space in order to fix the structure of the wound and provide good hygiene. For the EMTs, in the scene of the catastrophe, the 3D face imaging device provides them a good way to take care of the wound and may transfer the information to the doctors in the hospital. For example, the depth of the wound is useful information for a plastic surgery doctor to guide the EMTs to perform different types of wound dressing.

Conclusions: The application of multiple views 3D stereo-imaging technology to the medical and burn care field will help improve surgically and burn care accuracy, reduce operation times, and enhance patient safety. Therefore, this device has been applied to a variety of fields such as burn care and the plastic surgical or cosmetic medical fields. Particularly, in the medical and burn care field, various medical imaging devices have adopted the stereo-imaging technique, such as the stereo 3D camera and multiple views 3D camera, and have been developed and utilized clinically to improve surgical and burn care accuracy and patient safety.
\end{abstract}

Keywords: Burn Care, 3D Imaging, Multiple Views, Stereoscopic Vision, Visual Information, Visual System, Human Factor

\footnotetext{
* ftj@nut.edu.tw (alternative)wonderfulactioncom@gmail.com; +886- 921- 150529; https://ece.ntut.edu.tw/index.php?Lang=en
} 


\section{Introduction}

Close, multidisciplinary collaboration with burn experts is an essential strategy to achieve the best functional and esthetic outcomes in burn wound treatment. The specific origin and nature of the telemedicine requests for advice, transfer, or follow-up were collected, as were data relating to the demographics of the patients, the etiology, mechanism, and extent of the burn injury.

Management of minor burn injuries, where no specialized care is available, might be challenging. One concept to achieve a fast and timely result is the application of telemedicine. The impact of the 3D stereo matching image of communication in terms of reductions in admissions and clinic visits was assessed, and a cost analysis was undertaken. Feedback was also obtained from those health practitioners regularly using the service.

3D images and telemedicine are increasingly applied in developed settings to facilitate the transfer of information to and from burn surgeons across vast geographic areas. Multi-view 3D stereo matching image is a widely available and extremely user-friendly encrypted smartphone application that does not require the expensive physical and personnel infrastructure that characterizes many of these telemedicine and biometric systems.

Inexperience in burn wound assessment by referring physicians often results in over-triage or undertriage. In an effort to improve access to burn care in our region, we instituted a program of telemedicine evaluation of acute burns. The objective of this study was to assess and develop a simple multi-view 3D stereo matching protocol for initial assessment and follow-up of burn wounds to support the management of the burns treatment, which can be applied globally.

\subsection{Accurate Evaluation of Burn Wounds as Cancelable Biometric Identification}

Biometrics has long been used to recognize people, mainly in terms of their physiological characteristics, for various commercial applications ranging from surveillance and access control against potential impostors to smart interfaces [1,2].

These systems require reliable personal recognition schemes to either confirm or determine the identity of an individual requesting their services. The biometric methods, that are usually incorporated in such systems, can be categorized into physiological and behavioral $[3,4]$, depending on the type of used features.

However, despite their high recognition performance, static biometrics have been recently overwhelmed by the new generation of biometrics, which tends to cast light on more natural ways for recognizing people by analyzing dynamic behavioral traits

Biometric systems are based on the use of certain distinctive human traits, be they behavioral, physical, biological, physiological, psychological, or any combination of them. Biometric information (especially raw images) can expose sensitive information such as information about one's health origin and this information can then provide a basis for unjustified discrimination of the individual data subjects [5].

Accuracy recognition should be preserved (or degraded smoothly) when protected templates are involved. Many proponents of biometric systems claim that it is sufficient to store a compact representation of the biometric (template) rather than the raw data to ensure the privacy of individuals. They consider that template is not sensitive information because it does not allow the reconstruction of the initial signal. Recently, several research works showed that this reconstruction is possible. For example, fingerprint can, in fact, be reconstructed from a minutiae template [6,7].

In biometrics, 'recognition' may be used to refer to two distinct tasks. In the first one, that is called verification, an individual claim to be a certain user who has been previously registered(enrolled) into the system. It is also possible that the individual does not indicate his/her identity but there exists some additional information that allows to suppose it. In this case system operation is reduced to confirm or reject that a biometric sample belongs to the claimed identity. In the second task, identification, it is not available such prior information about an individual's identity, the system must determine which among all of the enrollees the subject is if any.

\subsection{Accurate Evaluation of Burn Wounds by 3D Stereo Matching System}

Uni-modal or 2D biometric systems have poor performance and accuracy, and over the last few decades, the multi-modal biometric systems have become very popular. The main objective of multibiometrics is to reduce one or more false accept rate, false reject rate, and failure to enroll rate.

Face Recognition (FR) is still considered as one of the most challenging problems in pattern recognition. The FR systems try to recognize the human face in video sequences as a $3 D$ object $[8,9]$, in unconstrained conditions, in comparison to the early attempts of $2 \mathrm{D}$ frontal faces in controlled conditions. Such 2D matching biometric system may not always meet performance requirements, may exclude large numbers of people, and may have been vulnerable to everyday changes and lesions of the 
biometric feature. Despite the effort spent on research today, there is not a single, clearly defined solution to the problem of Face Recognition, leaving it an open question.

Three dimensional (3D) image reconstruction is a procedure of creating a mathematical representation of a 3D object. There are various approaches for performing 3D imaging. On the smallest scale, interferometry is an extensively utilized technique for 3D surface reconstruction. With information extracted from the combined waves, interferometry can be used to inspect optical surfaces and provide a high precision mapping to a small fraction of a wavelength [10]. Another reliable non-contact 3D imaging technique is structured illumination. Having a calibrated projector-camera pair, a light pattern is projected onto the scene and imaged by the camera (or cameras). The structured illumination method uses the information from the distortion of the projected structured-light pattern to extract the 3D surface geometry.

In addition to these aforementioned techniques, stereo vision (also known as stereoscopic vision or stereopsis) is another extensively used technique in 3D imaging, which reconstructs a 3D object by deducing the spatial shape and position of the object through parallax between the corresponding pixels from different images of the object as observed from multiple viewpoints [11]. The principle of traditional stereo vision techniques is triangulation, in which the unique contours of the object can be determined with the photos taken from two unparalleled cameras [12]. Traditional stereo vision approaches rely on the correspondence between photo elements from two cameras which sometimes can be difficult to determine. It is also possible to extract 3D information from a single image, namely shape from shading [13], by utilizing assumptions of uniform surface reflectivity.

Advances in 3D imaging have allowed vision users to overcome some challenging inspection tasks. In the machine vision marketplace, 3D imaging continues to mature, tackling applications 2D imaging cannot. Improving 3D technologies cost-effectively simplifies a variety of inspection tasks. And it's taking machine vision inspection to the next level. And with inspection tasks in 3D space, which may include measurement or reconstruction, precision is even more essential than with most tasks in robotic guidance or bin picking. The combination of a 3D image with grayscale 'texture' and/or color information is somewhat new in the machine vision marketplace. Having this information can be critical to the identification, differentiation, location, or measurement of an object. A 3D image by itself has no grayscale or color content. Incorporating this content in a spatially correct way into a 3D image can have great benefits. In many cases, the 3D profile simply does not fully define the object or its features. Additionally, 3D enables users to measure the angle of the surface and planar features such as distance. Machine vision component suppliers are developing products that address common 3D imaging needs. However, existing technologies still suffer from minimizing the overall system size, require to achieve one of the modalities of 3D surface imaging techniques.

This required precise alignment of the cameras and pattern generators to be able to calculate, or triangulate, each point in the image to determine its distance from the camera. This process required a lot of computing power. Today's multicore embedded processor architectures make it possible to do these calculations at increasing resolutions and imaging rates, which enables in-line, real-time use of 3D image data for machine vision. What was once difficult to achieve can now be accomplished with off-the-shelf sensors and advanced software libraries, making it possible for systems integrators and machine makers to more easily integrate 3D measurements into their vision processes. When customers can just concentrate on how to mount a 3D sensor on their machine and trust that it will produce accurate, repeatable 3D point clouds, they're more likely to adopt the technology. When it comes to advanced machine vision technologies like 3D imaging, end-users expect the same benefits they always do - ease-of-use and cost-effectiveness. Vision product manufacturers are addressing these demands by developing targeted solutions that improve accuracy and reliability in numerous inspection tasks.

Implementing 3D surface imaging camera systems into miniaturized devices for a variety of 3D applications such as movement recognition, object sensing, and 3D endoscopy has received great attention over the past decade. Recently, various MEMS techniques enabled the fabrication of key optical elements for 3D surface imaging with compact size, reasonable cost, and high yield.

New trends in biometrics are inclined to adapt both the identification and verification process to mobile devices in order to provide realistic scenarios and applications with a more secure frame. In fact, upcoming applications related to electronic commerce are demanding more trustworthy and reliable techniques to ensure their operations and transactions [14], for instance.

In general, multimodal or multi-view 3D stereo matching systems are considered to provide an excellent solution to a series of recognition problems. Unimodal or 2D matching systems are more vulnerable to theft attempts since an attacker can easily gain access by stealing or bypassing a single biometric feature. 


\subsection{New Virtual Tool for Accurate Evaluation of Burn Wounds by Combining Biological Data with 3D Stereo Matching System}

Imaging cameras in various fields have been used to capture three-dimensional (3D) objects in reality into two- dimensional (2D) images without depth information with conventional image sensors. However, this deficiency of 3D information limits the perception and intensifies the confusion for understanding the real world. For decades, numerous technical efforts have been made in research, development, and commercialization for 3D surface imaging technologies in many applications.

This study provides a system (method and apparatus) for creating photorealistic multi-view 3D stereo matching models of burn wounds from a plurality of stereo images obtained from the stereo 3D camera and optional mono- or bi-ocular cameras. The cameras may be handheld, mounted on a manipulator, or a positioning device. The system detects and tracks features in image sequences and self-references the stereo camera by matching the features to a database to track the burn wounds while building the database simultaneously. Individual stereo pairs are processed to compute dense 3D data representing the scene and are transformed into a common reference and fused together. The resulting 3D stereo data is represented as point clouds, surfaces, or volumes. The present invention also provides a system (method and apparatus) for enhancing 3D models of burn wounds by registering information from additional sensors to improve model fidelity or to augment it with supplementary information by using a light pattern projector.

\section{Related Work}

\subsection{Biometric performances of Burn Biological Data}

In biometric recognition systems, however, almost never the data captured from a subject (nor the feature vectors obtained from them) are identical to the previous ones [17]. The reasons for these variations are manifold and include the following:

A. Imperfections in the capture process that create alterations (e.g., noise) in the data;

B. Physical changes in the capture environment (e.g., changes in lighting and degradation of the sensors used); and

C. Inevitable changes over time in an individual's biometric traits.

D. As a result of the unrepeatability of biometric samples, the process of biometric recognition cannot be deterministic and it must be based on the stochastic behavior of samples. In this way, rather than flatly asserting correspondence between a biometric sample and the corresponding model, biometric systems only permit the assertion that this correspondence has a certain probability of being true.

The ideal biological data for biometrics has the following five characteristics [15,16]:

a. Distinctive: the biological data differs from one person to another.

b. Repeatable: the biological data remains constant over a long period.

c. Accessible: it is easy to view the biological data.

d. Acceptable: it is not objectionable to show the biological data.

e. Universal: all people possess biological data.

From different viewpoints, the five characteristics are associated with the potential problems and limitations of burn biometric identification.

\subsection{Problems of burn identification}

The three main problems are as follows:

Problem 1: The biological data cannot be replaced.

For instance, if a user's fingers are lost, or if fingerprint information is stolen, the user cannot use a fingerprint identification system. This problem is related to characteristics (b) and (d).

Problem 2: Users are specified only from the biological data.

As biological data is information linked directly with individuals, if biological data is leaked, the user can be specified using only the leaked biological data. This problem is related to characteristic (a).

Problem 3: The biological data can be collected without the consent of the user.

In general, because biological features are exposed on the surface of the body, such as the face, fingerprints, and iris, it is difficult to keep these features concealed from others. This problem is related to characteristics (c) and (e).

Due to these problems, current biometric identification systems have a major vulnerability: spoofing $[18,19,20]$.

While multi- and dynamic- traits may be used in an isolated manner by biometric recognition systems, experience has shown that results from biometric systems analyzing a single trait are often insufficiently reliable, precise, and stable to meet specific performance demands $[21,22]$. In order to dynamic system 
performance closer to the level expected by the burn injury, therefore, novel biometric recognition systems have been designed to take advantage of taking multiple traits into account.

\section{Proposed Methodology}

In this study, the development of systems that integrate more than one biometrics is an emerging trend, since it has been seen that true multimodal and multi-view 3D stereo matching biometric systems, that capture a number of unrelated biometrics indicators, have significant advantages over $2 \mathrm{D}$ or unimodal ones. Specifically, most of the aforementioned limitations can be addressed by deploying multi-view 3D stereo matching systems that integrate the evidence presented by multiple sources of information. This allows the integration of two or more types of recognition systems, in order to meet stringent performance requirements. Last but not least, a major issue of biometric systems is the protection of the sensitive biometric data that are stored in the database, so as to prevent unauthorized and malicious use.

Scanning Process

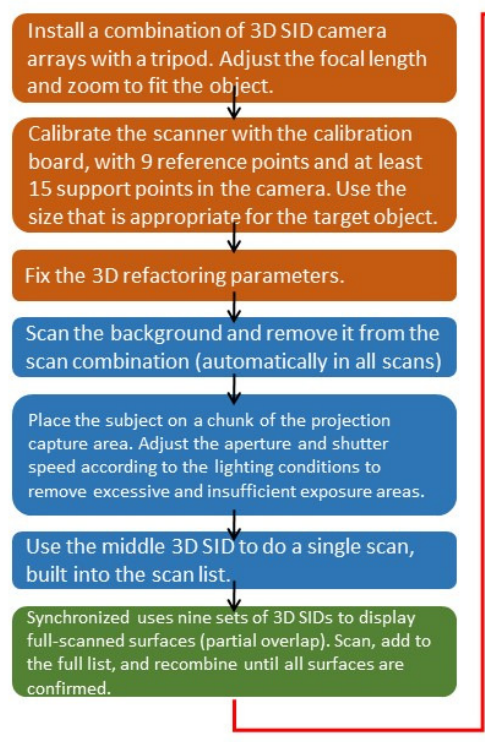

3D Facial Specification Database Modeling Process

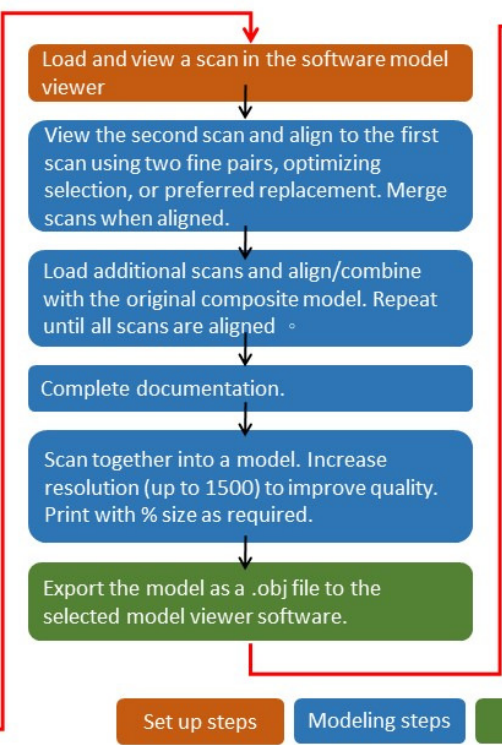

3D Printing Process

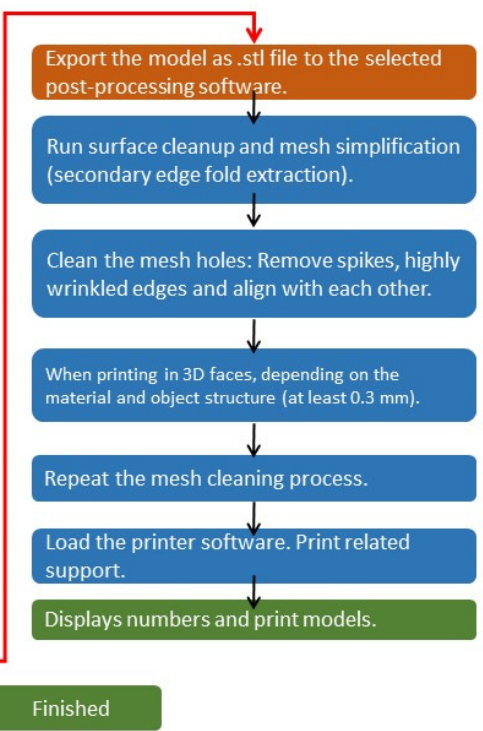

\subsection{Burn Wounds Detection System: Enrollment and Verification Step}

A biometric system can be viewed as a signal detection system with a pattern recognition architecture that senses a raw biometric signal, processes this signal to extract a salient set of features called biometric identifier or template, and compares these features against the ones stored in the database $[23,24]$. The uniqueness of biometric characteristics allows an intruder to link users between different databases, enabling violations as tracking and profiling individuals.

More precisely, all biometric systems involve two steps. Enrollment step: biometric data (face) are captured, transformed into a template linked to the individual, and stored as a reference. Verification step: a new template is issued from a new capture, and compared to the stored reference template.

\subsection{Burn Wounds Detection System: Conventional 3D Surface Imaging Techniques}

Recently, market demand for miniaturized 3D optical imaging modules has been remarkably increased since smart devices, wearable devices, or multifunctional imaging devices have attracted both customer and developer's interest. However, regardless of the high technological maturity of 3D surface imaging techniques, miniaturized optical key elements for 3D surface imaging is required to be packaged into compact imaging systems, such as multifunctional cameras in smartphones and 3D endoscopic catheter.

Numerous 3D surface imaging techniques have been developed using stereoscopic vision, structured light, time-of-flight (ToF), interferometry, holographic imaging, and so on. Stereoscopic vision, structured light, and ToF are considered as three major techniques and actively investigated because they provide higher resolution, high speed, and intuitive applicable principle compared to others. 
The stereoscopic vision method utilizes two or multiple image sensors and concurrently captures the same scene from different viewpoints. After the rectification process of stereo images, depth information can be calculated by comparing the image pixel disparities of rectified stereo images.

However, the stereoscopic vision method has already been widely adopted in various 3D imaging commercialized products such as 3D movie recorder [25] and 3D medical endoscopes [26] with significant advantages in low-cost, intuitive principle, and compact configuration.

\subsection{Burn Wounds Detection System: Biometric fusion}

Biometric fusion is composed of a set of mono-biometric sub processes that work in parallel to operate the data obtained from distinct sources. The possible responses in the literature to two points allow establishing diverse characterizations of data fusion systems defined as fusion levels $[27,28,29]$.

The first point at which data fusion may be carried out is at the sample level, that means immediately following sample capture by system sensors. This type of fusion is possible in multi-sensor, multiinstance, and multi-sample systems and may be obtained by following a particular sample fusion method. The form that this method takes in each case depends on the type of biometric trait being utilized. While fusion may range from a simple concatenation of the digitalized sample data sequence to more complex operations between multiple sequences, but it is almost always carried out for the same reason: to eliminate as many negative effects as possible associated with the noise encrusted in the data samples during capture. Once the fused sample has been generated, it may be used by the system for feature extraction.

The second point at which data may be combined is immediately following the feature extraction. At the feature level, vectors derived from the different sources are combined, yielding a single, fused vector. Another alternative is the fusion of scores obtained following the matching of different sample data against corresponding models. The new score resulting from this fusion is then used by the system to reach the final decision. This sort of fusion is normally carried out according to mathematic classification algorithms ranging in type from decision trees to techniques from the field of artificial intelligence, the latter of which offering learning capabilities and requiring prior training.

\subsection{Burn Wounds Detection System: Data Sources}

In order to respond to the first question of the previous paragraph regarding multiple data sources, the following specific questions must also be considered [27].

1. How many sensors are to be utilized? In the construction of multi-view 3D stereo matching systems, different sensors (each with distinct performances) are used in order to capture multiple samples of a single burn trait. In one example of this sort of multimodal and multi-sensor systems, simultaneous photographs are captured of a subject's face using visible light cameras.

2. How many instances of the same burn trait are to be captured? Patient beings can present multiple versions of particular burn traits. As a result and with a schema similar to that of multi-sensor systems, multi-view 3D stereo matching systems are designed to capture various instances of the same burn trait.

3. How many times is an instance of a particular burn trait to be captured? Using a single sensor and a single instance of a particular burn trait, it is nevertheless possible to obtain distinct samples of that instance under different conditions (e.g., images taken of a trait instance from different angles). These multi-sample systems may also be represented by a schema similar to that of multi-view 3D stereo matching systems.

4. How many different burn traits are to be captured? Burn wound recognition systems may be designed to analyze unimodal systems or multimodal and multi-view 3D stereo matching systems.

5. How many distinct feature extraction algorithms are to be utilized in the processing of the burn samples? Multi-algorithm systems are designed to use various algorithms for feature extraction from burn samples. In this study, the use of different extraction algorithms may allow the system to emphasize different biometric features of interest and produce different feature vectors for each.

6. Against how many types of patterns and using how many methods are the feature vectors to be matched? Multi-view 3D stereo matching systems are burn-recognition systems that allow match the feature vectors against various types of models and using multiple techniques.

7. Finally, it is also possible to construct hybrid systems of even greater complexity that incorporate more than one type of the multiple data sources discussed above. 


\subsection{Photorealistic Multi-View 3D Stereo Matching Systems: New Virtual Tool for Accurate Evaluation of Burn Wounds}

The preliminary invention, the development of systems provides a method and apparatus for producing photorealistic multi-view 3D stereo matching systems of initial assessment and follow-up of burn wounds from a plurality of stereo images obtained from the stereo 3D camera. The cameras may be handheld, mounted on a manipulator, or a positioning device. In one aspect of the invention there is provided a method of producing a three-dimensional (3D) stereo model of an burn wounds, comprising the steps of: a) acquiring a plurality of stereo images of burn wounds from at least one stereo camera; b) detecting $3 \mathrm{D}$ features in the stereo images and computing a 3D position and descriptor for each detected 3D feature in a database; c) computing relative motion of the at least one stereo camera with respect to burn wounds by matching the detected 3D features in the stereo images with 3D features stored in the database using descriptors of said 3D stereo features; d) computing dense 3D stereo data sets, representative of the object, from at least one range sensing device; e) transforming the computed dense 3D stereo data from step d) into a selected coordinate frame of reference using the computed burn wounds from step c) to give transformed dense 3D data in the selected coordinate frame of reference; and f) storing the transformed dense 3D stereo data, and producing a multi-view 3D stereo matching model of the burn wounds from the stored transformed dense 3D data suitable for visualization, analysis or post-processing.

Moreover, the face can be acquired without constraints in orientation, distance to the camera, or illumination, since the proposed technique within this paper is invariant to previous changes. This property provides an increase in the acceptance of the biometric technique by the final user, together with the fact and the like is required for image acquisition.

In contrast, such a lack of constraints in acquisition demands a more challenging solution in relation to segmentation and feature extraction. The former operation must be able to isolate the head completely from the background, regardless of what is behind the head.

In the case of feature extraction, the template must be independent of which head is considered for identification and invariant to changes in orientation, position, distance to the camera, and the like. In addition, the proposed template considers face widths and lengths, and, besides, information from light is considered, instead of global features from the whole head.

\section{Result Analysis and Discussion}

\subsection{Results}

We have achieved burn wound resolution, in patients, with no scarring and only minor discoloration. A simple, reproducible treatment protocol was designed to include dressing changes and additional outpatient visits.

Throughout the entire burn care process, telemedicine has been used not only to provide an expert advice from distance but also to help establish and maintain the doctor-patient relationship, to keep patients in contact with their families, and to help educate and consult the medical personal physically present in the application of the multi-view 3D stereo matching image.

Queries related to the full spectrum of burn care, including emergency management and stabilization, triage and transfer, the need for escharotomy, fluid resuscitation, wound care, the timing, and nature of the surgical intervention, as well as follow-up and rehabilitation.

The proposed system has been tested with three databases collected in different burn wounds. The first database was created to evaluate the proposed algorithm in terms of detection accuracy, containing samples of individuals from a population of different ages and gender. The segmentation algorithm is exclusively tested by images in the second database, a collection of synthetic face images, based on the first database, but with different environments, so that real scenarios can be simulated. Finally, the third database was collected to evaluate to what extent segmentation and feature extraction algorithms were invariant to different degrees, distance to the camera, and rotation.

After a patient presents the burn trait which the multi-view 3D stereo matching system's sensor is designed to process, in the first stage (i.e., capturing sample), a burn biometric sample is obtained by the sensor and processed by the system to eliminate noise, emphasize certain features of interest and, in general, prepare the sample for the following stage of the process. In the next step (i.e., feature extraction), characteristic parameters of the sample are quantified and a feature vector that groups them is obtained. Following quantification, the system proceeds to match the feature vector (i.e., model matching) against others captured during the training phase that corresponds to the individual whose identity is being claimed. 


\subsection{The following specific questions must also be considered}

1. Efficiency: For large shape collections, it is inefficient to sequentially match all objects in the database with the query object. Because retrieval should be fast, efficient indexing search structures are needed to support efficient retrieval. Since for query by example, the shape descriptor is computed online, it is reasonable to require that the shape descriptor computation is fast enough for interactive querying.

2. Discriminative Power: A shape descriptor should capture properties that discriminate objects well. However, the judgment of the similarity of the shapes of two 3D objects is somewhat subjective, depending on the user preference or the application at hand. E.g. for solid modeling applications often topology properties such as the numbers of holes in a model are more important than minor differences in shapes. On the contrary, if a user searches for models looking visually similar to the existence of a small hole in the model, it may be of no importance to the user.

3. Partial Matching: In contrast to global shape matching, partial matching finds a shape of which a part is similar to a part of another shape. Partial matching can be applied if 3D shape models are not complete, e.g. for objects obtained by laser scanning from one or two directions only. Another application is the search for "3D scenes" containing an instance of the query object. Also, this feature can potentially give the user flexibility towards the matching problem, if parts of interest of an object can be selected or weighted by the user.

4. Robustness: It is often desirable that a shape descriptor is insensitive to noise and small extra features, and robust against arbitrary topological degeneracies, e.g. if it is obtained by laser scanning. Also, if a model is given in multiple levels-of-detail, representations of different levels should not differ significantly from the original model.

\subsection{Photorealistic Multi-View 3D Stereo Matching Systems: Applications}

The photorealistic multi-view 3D stereo method of producing a 3D model of initial assessment and follow-up of burn wounds, comprising the steps of: a) acquiring a plurality of stereo images of an environment or an object from at least one stereo camera, the stereo camera having at least two individual image capture means where there is an overlap between images captured by said at least two individual image capture means; b) detecting 3D features in the stereo images and computing a 3D position and descriptor for each detected 3D feature in a database; c) computing relative burn wounds of the at least one stereo camera with respect to the object by matching the detected 3D features in the stereo images with 3D features stored in the database using descriptors of said 3D features; d) computing dense 3D data sets, representative of the object, from at least one range sensing device; e) transforming the computed dense 3D data from step d) into a selected coordinate frame of reference using the computed relative burn wounds from step c) to give transformed dense 3D stereo data in the selected coordinate frame of reference; and f) storing the transformed dense 3D stereo data, and producing a photorealistic multi-view 3D stereo model of the object from the stored transformed dense 3D data suitable for visualization, analysis or post-processing.

Another consideration when calculating the cost of $3 \mathrm{D}$ rendering is that $3 \mathrm{D}$ imagery has been shown to both increase conversions and reduce returns on e-commerce sites. The upfront investment, then, can be recovered as a brand sells more and receives fewer returns thanks to its more accurate images.

Finally, the files created during the $3 \mathrm{D}$ rendering process position a brand to create more rich visual content in the future, including augmented reality (AR) and virtual reality (VR). While both are in their relative infancy today, they're becoming increasingly popular in various settings. Brands that are positioned to add these features to their sites will be the best prepared to compete tomorrow in ecommerce, entertainment, and elsewhere.

\subsection{Discussion}

The initial evaluation of burns and highlights the areas, correct estimation of the severity of burns is important to obtain the right treatment of the patient and to avoid over- and under-triage.

Estimation of acute burn injuries is known to be difficult, but to initiate relevant treatment, a thorough and precise evaluation is essential. The initial estimation relates to the size, depth, and localization of the injury as well as several concomitant factors. Advanced technology has increased the use of telemedicine, biometric system, and Information Technology (IT) in treating or rehabilitating diseases. Incorporating multi-view 3D stereo matching image technology into the daily processes of burn care has significantly improved the quality of burn care referrals to specialist burn services. The initial treatment and the types of burn injuries needing referral to specialist care are summarized, which most often cause difficulties. 
Specifically, the 3D stereo matching image has contributed to reductions in unnecessary referrals and outpatient visits, facilitated opportunities for continuing medical education, improved the care of major burn injuries through more effective prehospital communication, and enabled greater allocation of scarce specialist resources at the burn center. This study motivates for the wider application of 3D stereo matching image for burn care referrals, in both developed and developing countries, especially to support the treatment of burn victims in a low-income country that receives reconstructive surgery. Constructing a 3D model usually needs professional graphic technology and scanning equipment. This often in vast time consumption and high costing budgets. To solve the problem of high cost and complicated process, a simple, fast, and low-cost method is proposed. Firstly, take images with 360degree panorama of the object that is going to be modeled. Then update the image set to the database in the cloud. With the powerful cloud technology and computing power, the image set will be transformed into a realistic 3D model that can be downloaded by clients. The professional modeling technique of users is no need for this method. To get a realistic 3D model all we need to do is just taking panoramic images of the object and finish a few steps of simple settings.

\section{Conclusion}

Acute evaluation of burn patients can be performed accurately by multi-view 3D stereo matching images. This can reduce under-triage or over-triage for transport, improve resource utilization, and both enhance and extend burn center expertise to many communities by telemedicine at low cost.

Implementation of a multi-view 3D stereo matching protocol in 5G telemedicine allows for easy access to burn consultations, helps multidisciplinary collaboration, eases follow-ups, and shortens specialists' consult wait times. The real-time evaluation provides fast and flexible treatment, without long-distance travel, for patients and their families. 3D stereo image in $5 \mathrm{G}$ telemedicine increases the frequency of follow-up, contributes to the esthetic outcome, and together with improved costeffectiveness is beneficial for both the patient and healthcare system.

There is a growing body of evidence highlighting the benefits of mobile health in terms of costeffectiveness, efficiency, and patient satisfaction. These benefits have been further enhanced through the development of Instant Messaging (IM) applications (apps) that enable the transmission of images and text messages. This study presents the details of how this multi-view 3D stereo matching process has been conducted to date, on what was learned from this process, and on issues that should be considered to improve this process in the future.

This study reports on the utilization of multi-view 3D stereo matching images for initial assessment and follow-up of burn wounds to support the management of the burns treatment.

The authors plan to create an instructional 3D stereo matching image and post them on the biometric system to aid clinical workers providing similar treatment during the acute care and rehabilitation process and also to support eLearning in many situations where it otherwise is not possible to use multiview 3D stereo conferencing to establish real-time contact between doctors at the local site and remote specialists.

\section{References}

[1] F.A. Qazi, "A survey of biometric authentication systems, security and management", 2004, pp. 6167.

[2] Q. Xiao, "Security issues in biometric authentication", presented in Information Assurance Workshop publication, 2005, pp.8-13.

[3] A.K. Jian, A. Ross, S. Prabhakar, "An Introduction to Biometric Recognition", in Journal of IEEE Trans. Circuits Syst. Video Technol., Vol.14, No.1, 2004, pp.4-20.

[4] A. Jian, K. Nandakumar, A. Ross, "Score normalization in multimodal biometric systems", in Journal of Pattern recognition, Vol.38, No.12, 2004, pp.2270-2285.

[5] E. Mordini, A. Massari, "Body, biometrics and identity", in Journal of Bioethics, Vol.22, No.9, 2008, pp.488-494.

[6] R. Cappelli, A. Lumini, D. Maio, D. Maltoni, "Fingerprint image reconstruction from standard templates", in Journal of IEEE Transactions on Pattern Analysis and Machine Intelligence, Vol.29, No.9, 2007, pp.1489-1503.

[7] J. Feng, A. Jain, "Fm model based fingerprint reconstruction from minutiae template", presented at International conference on Biometrics, 2009.

[8] K.I. Chang, K.W. Bowyer, P.J. Flynn, "Face recognition using 2D and 3D facial data", presented in ACM Workshop publication on Multimodal User Authentication, 2003, pp.25-32.

[9] K.I. Chang, K.W. Bowyer, P.J. Flynn, "An evaluation of multimodal 2D+3D face biometrics", in Journal of IEEE Trans. Pattern Anal. Mach. Intell., Vol.27, 2005, pp.619-624. 
[10]P. Hariharan, Basics of Interferometry, Boston MA, USA, Academic Press, 2010.

[11]L. locchi, K. Konolige , "A multiresolution stereo vision system for mobile robots", presented in New Trends in Robotics Research publication, Padova Italy, 1998, pp. 317-321.

[12] E. Keppel, "Approximating complex surfaces by triangulation of contour lines", presented in IBM J. Res. and Dev., Vol.19, 1975, pp.2-11.

[13]B. K. Horn, M.J. Brooks, Shape from Shading, Cambridge MA, USA, MIT Press, 1989.

[14]D. Van Thanh, "Security issues in mobile ecommerce", presented in Database and Expert Systems 11th International Workshop publication, 2000, pp.412-425.

[15]J. Wayman, "National Biometric Test Center Collected Works 1997-2000", 2000, pp. 1-3.

[16]J. Ashbourn, "Biometrics: Advanced Identity Verification, The Complete Guide, Springer-Verlag", 2000.

[17]S. Ross, K. Nandakumar, A.K. Jain, Handbook of Multibiometrics, Springer Publishers, 2006.

[18] A.K. Jain, S. Pankanti, S. Prabhakar, L. Hong, A. Ross, J.L. Wayman, "Biometrics: A Grand Challenge, Proceedings", presented in International Conference publication on Pattern Recognition, 2004, pp.935-942.

[19] H. Matsumoto, T. Matsumoto, "Artifact-metric Systems", presented in Technical Report publication of the Institute of Electronics Information and Communication Engineers, 2000, Vol.59, pp.7-14.

[20]T. Matsumoto, "Biometric Authentication Systems: Vulnerability of Biometric Authentication - On the Issue of Physiological Spoofing", in Magazine of Information Processing Society of Japan, Vol.47, No.6, 2006, pp.589-594.

[21]S. Ross, K. Nandakumar, A.K. Jain, Handbook of Multibiometrics, Springer Publishers, 2006.

[22] A. Ross, N. Poh, Multibiometric Systems: Overview, Case Studies and Open Issues, in Handbook of Remote Biometrics for Surveillance and Security, Springer, 2009.

[23] A. Jain, K. Nandakumar,A. Nagar, Biometric template security, Signal Process, 2008.

[24]A. Jain, A. Ross, S. Pankanti , "Biometrics: A tool for information security", in Journal of IEEE ransactions on Information Forensics and Security, Vol.1, No.2, 2006, pp.125-143.

[25]B. Mendiburu, Stereoscopic vision and 3D cinematography, Focal Press, 2009.

[26] KW. Nam, J. Park, IY Kim, KG Kim, "Application of stereo-imaging technology to medical field", in Journal of Healthc Inf Res, Vol.18, No.3, 2012, pp.158-163.

[27]A. Ross, " An Introduction to Multibiometrics, Proc.", presented at 15th European Signal Processing Conference, Poznan Poland, 2007.

[28] A. Ross, A. Rattani, M. Tistarelli, S. Prabhakar, " Exploiting the Doddington Zoo Effect in Biometric Fusion, Proc.", in Journal of IEEE International Conference on Biometrics: Theory, Applications and Systems, 2009.

[29] H.G. Kumar, M. Imran, " Research Avenues in Multimodal Biometrics", in Journal of RTIPPR, Vol.1, 2010, pp.1-8 